\title{
Saw-type shock fronts in several HII regions of southern hemisphere
}

\author{
A. L. Gyulbudaghian* \\ NAS RA V. Ambartsumian Byurakan Astrophysical Observatory (BAO), Armenia
}

\begin{abstract}
Radiation shock fronts at the heads of non adiabatic cooling jets are considered. Decay of these shock fronts leads to the origin of groups of $\mathrm{HH}$ objects, and of groups of bright condensations in the jets expelled from the nuclei of galaxies, e.g. from the nucleus of NGC5128. Several examples of saw-type radiation shock fronts, found in the Southern Hemisphere, are presented.
\end{abstract}

Keywords: shock front: HH objects: galaxy NGC5128

\section{Introduction}

Highly-collimated outflows, or jets, are rather common among young stars and active galaxies. Much work has been devoted to adiabatic jets, though there has been little attention to nonadiabatic or "cooling" jets. Radiation cooling due to collisional excitation and recombination can be important to the energy budget of jets associated with young stellar objects and $\mathrm{HH}$ objects. In Blondin et al. (1990) is presented detailed investigation of cooling jets, using numerical modeling for cylindrically symmetric non adiabatic jets. Stellar jets have been observed in many regions of low mass star formation. These jets are detected through low excitation line emission, e.g., [SII], which is believed to be originated in gas that has been heated in oblique shocks within a supersonic beam. These jets are well collimated, with opening angles of only a few degrees, and highly supersonic, with a Mach number of $M \sim 20$ for an assumed jet temperature of $10^{4} \mathrm{~K}$ (Blondin et al., 1990). These outflows are intimately connected with $\mathrm{HH}$ objects. Many, if not most, $\mathrm{HH}$ objects are in some way related to jets. Giving density $\left(10-100 \mathrm{~cm}^{-3}\right)$ and shock front velocity $(50-200 \mathrm{~km} / \mathrm{s})$, which are typical for stellar jets and HH objects, in Blondin et al. (1990) was received, that the cooling time of gas, heating by shock front, often is less than the dynamic age of these objects, hence they have a conclusion that suggestion about adiabatic gas is invalid, it is necessary to consider the radiation shock fronts.

\section{Radiation shock fronts}

Interpretation of $\mathrm{HH}$ objects using radiation fronts, as was suggested by Schwartz (Schwartz, 1975), gave good coincidence with observed lines ratio. Several HH objects are seen as evident bow shock fronts at the top of bright stellar jets. The calculations show (Blondin et al., 1989), that some of these complicating, often divided in cloudlets, emission objects can be associated with active surface in the head of stellar jets. The dynamic envelope at the head of jet often is transforming into several small condensations of high density, creating a group of emission objects, resembling a group of $\mathrm{HH}$ objects. When the envelope begins to be destroyed, the condensations, which are actually formed out of the same envelope, continue to be associated with the active surface of the jet. In Blondin et al. (1989) was obtained, that the evolution can lead to the separate emission condensations, which show a rather big difference in their own velocities, though they were formed from the same envelope. The similar mechanism of disintegration of the envelope can be due to the Rayleigh-Taylor instability, as a

*agyulb@bao.sci.am 
result of propagation of the jet through the gas medium having a high density gradient. It is possible to suppose that the jet at the edge of $\mathrm{HH} 2$ recently has reached the region of low density, that is why the envelope of the jet was destroyed, creating several condensations with a high difference in their velocities (these condensations are forming now the object HH 2 itself) (Blondin et al., 1989). In Gyul'Budagyan (1984) we have considered the objects HH 1, HH 2 and HH 39, which are representing groups of condensations (the velocities of condensations in each group are rather different). We have received there, that in the center of inertia system the velocities of condensations in the same group originate from one center. This is in favor of mentioned above theory of formation of $\mathrm{HH}$ objects by decay of the envelope of the jet. If we suppose, that $\mathrm{HH}$ objects were obtained by decay of some bodies, then the values of velocities of these bodies, as was shown in Gyul'Budagyan (1984), are rather close to each other: $240 \mathrm{~km} / \mathrm{s}$ for HH $1,175 \mathrm{~km} / \mathrm{s}$ for HH 2 and $218 \mathrm{~km} / \mathrm{s}$ for HH39 (these velocities are close to the given above velocity of maximal shock front, $\sim 200 \mathrm{~km} / \mathrm{s}$ ). The condensations mean velocities in the center of inertia system are also close to each other: $63 \mathrm{~km} / \mathrm{s}$ for $\mathrm{HH} 1,71 \mathrm{~km} / \mathrm{s}$ for $\mathrm{H}, 2$ and $114 \mathrm{~km} / \mathrm{s}$ for HH 39 (Gyul'Budagyan, 1984). A case of existence of two differing by $200 \mathrm{~km} / \mathrm{s}$ radial velocities in the object $\mathrm{HH} 46$, was described in Dopita (1978). It is possible, that the object HH 46 consists of two condensations, which have not yet divided.

In the jet, ejected from the nucleus of the galaxy NGC5128, a group of emission condensations with remarkable difference in velocities is situated (Osmer, 1978). It is possible to imagine, that this group has also formed by decay of ionization front in the head of supersonic jet, ejected from the nucleus of the galaxy.

The morphology of supersonic jets (adiabatic or cooling) can be divided into several structures:the supersonic beam, a cocoon of shocked gas of the beam, shocked ambient gas and a leading bow shock. The term "jet" is used rather loosely, usually for describing of the whole structure, but sometimes for only the supersonic gas (Blondin et al., 1990). The prevailing property of the cooling gas (it is the surprising result of simulations) is the dynamic instability of dense envelope in the head of the jet. Fig.4 in Blondin et al. (1990) shows typical decay of the jet envelope due to dynamic instability. Inclined jet is entering into the beam with high density in the cocoon, forming a ring of more dense gas around the edges of the beam. It makes the cooling distance behind the jet shock front less at the edge of the beam, than in its center. Rapid cooling of ambient gas leads to the formation of a ring of dense matter in the post shock region, forming a hole, through which more tenuous, cooled gas is pushed away by the post shock pressure. When this hole is compressed, it behaves as de Laval nozzle, accelerating hot gas up to ultra sound velocities. During the process of acceleration of the envelope, Rayleigh-Taylor instabilities begin to grow, forming irregularities in the envelope (see Fig.4 in Blondin et al. (1990)). In Fig.4 from Blondin et al. (1990) it is possible to see different profiles of jet head, in Fig.4d that profile is saw-type. As is mentioned in Blondin et al. (1990), the age of such a profile is about 1500 years.

\section{Saw-type shocks, found in Southern Hemisphere}

During systematic inspection of high quality film copies of the ESO B, R, ESO/SRC J and EJ plates of Southern Hemisphere, we have found several saw-type shocks indifferent H II regions of Southern Hemisphere. In Table 1 the data on these shock fronts are summarized. In Table 1 in the first column the number of objects is given; in columns 2 and 3 - the coordinates of objects; in column 4 - the names of the stars, which are in the same distance as the objects;in column 5 - the distance to these stars, taken from (Gaia, 2018); in column 6 - the width of saw-type shock fronts.

From Table 1 we can conclude, that the smallest width is at object N 3, which is situated near the star with spectrum GO, and the biggest width is at the object 4, which is situated near the star with spectrum O5. If we suppose, that the shock fronts were ejected from the stars from Table 1, we can conclude, that the width of the shock front depends on the spectra - if the spectrum of the star is earlier, the width of the shock front is broader. In Fig.1 - Fig.4 we give the DSS2 R images of objects from Table 1. 
Table 1. Saw-Type shock fronts, found in southern hemisphere

\begin{tabular}{ccccccc}
\hline NN & $\alpha(2000)$ & $\delta(2000)$ & Star & Spectrs & Distance $(\mathrm{pc})$ & Width $(\mathrm{pc})$ \\
\hline 1 & 072955 & -465355 & HD61391 & B9 & 140 & 2.1 \\
2 & 073103 & -482500 & & & 0.92 & \\
3 & 170052 & -381857 & HD153330 & GO & 100 & 0.09 \\
4 & 170256 & -374150 & SAO208356 & O5 & 1280 & 4,5 \\
5 & 170303 & -375419 & & & & 1.1 \\
6 & 171833 & -383333 & HD156232 & AOIV & 270 & 0.48 \\
\hline
\end{tabular}

\section{References}

Blondin J. M., Konigl A., Fryxell B. A., 1989, ApJL, 337, L37

Blondin J. M., Fryxell B. A., Konigl A., 1990, ApJ, 360, 370

Dopita M. A., 1978, ApJS, 37, 117

Gyul'Budagyan A. L., 1984, Astrophysics, 20, 75

Osmer P. S., 1978, ApJL, 226, L79

Schwartz R. D., 1975, ApJ, 195, 631 


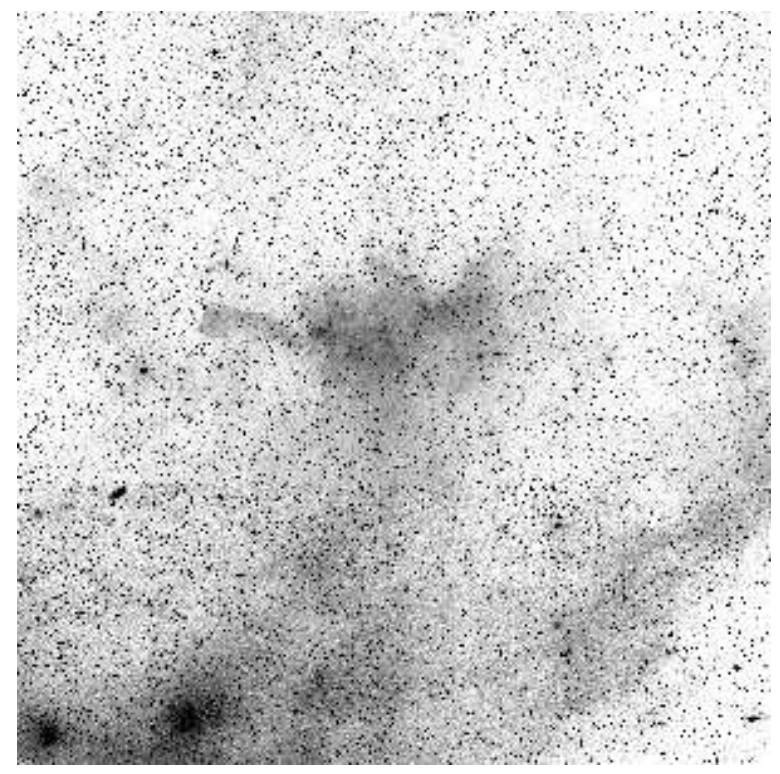

Figure 1. DSS2 R image, including the objects $\mathrm{N} 1$ and $\mathrm{N} 2$ from Table 1 . Object $\mathrm{N} 1$ is in the center of image, object N 2 is near the southern edge of image. North is to the top, south to the bottom. The sizes of image are $3^{\circ} \times 3^{\circ}$.

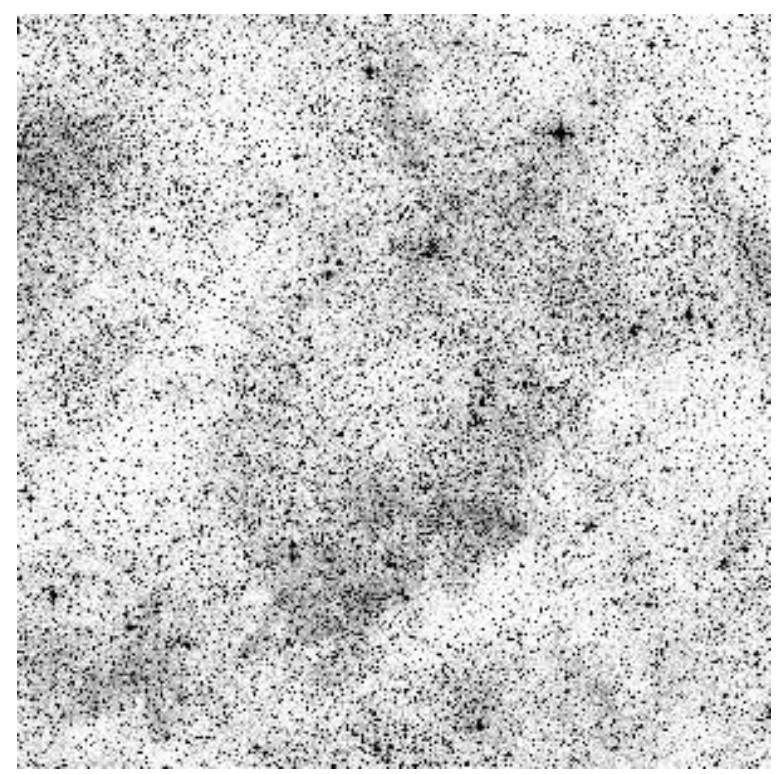

Figure 2. DSS2 R image of the object N 3 from Table 1. The object is situated a little to the south from the center. The sizes of image are $0.8^{\circ} \times 0.8^{\circ}$. 


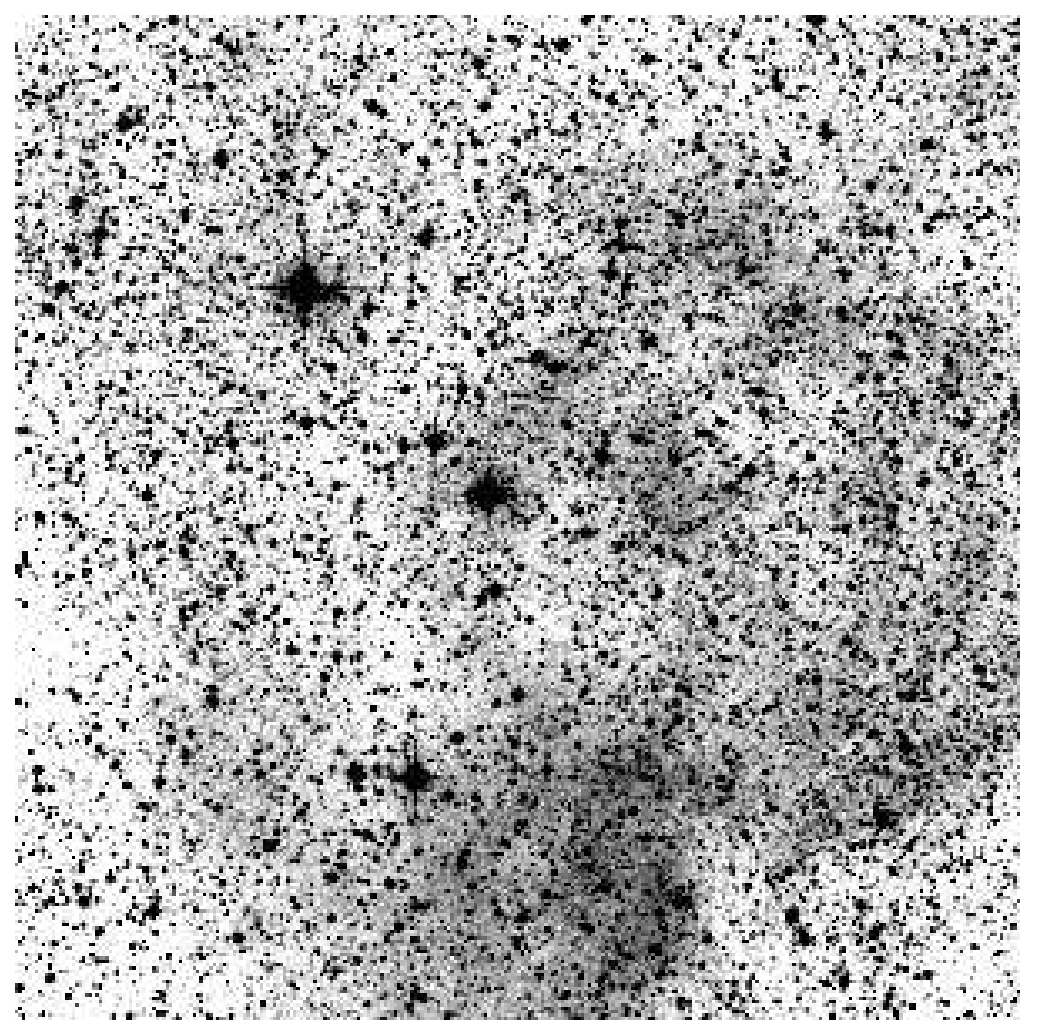

Figure 3. DSS2 R image of the object N 4 from Table 1. The object is situated a little to the SW from the center. The sizes of image are $0.4^{\circ} \times 0.4^{\circ}$.

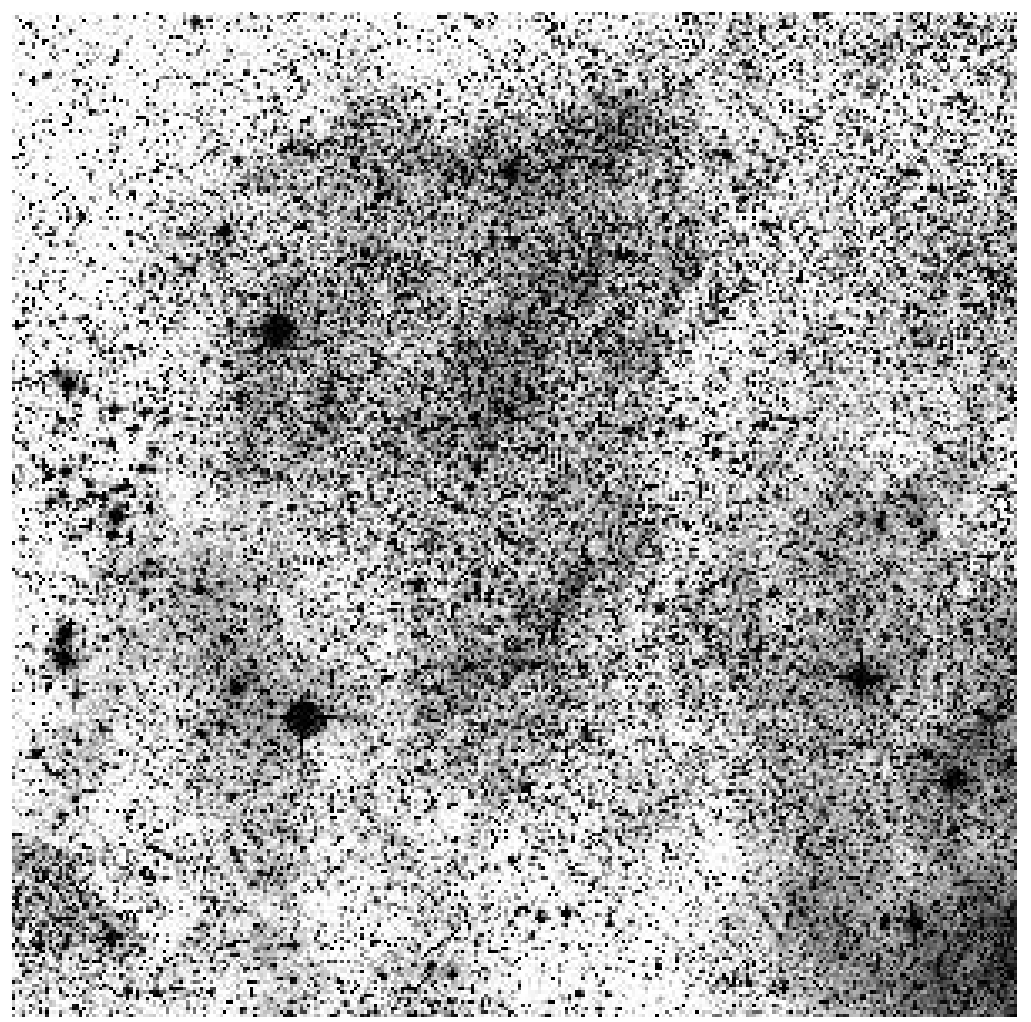

Figure 4. DSS2 R image of the objects N 5 and N6. The object N 5 is situated to the $\mathrm{N}$ from the center, near the edge of the image. The object $\mathrm{N} 6$ is also situated to the $\mathrm{N}$ from the center, but closer to the center than the object $\mathrm{N} 6$. The sizes of image are $0.8^{\circ} \times 0.8^{\circ}$. 